\title{
Disclosure of the Mechanisms of Metabolic Disorders in Highly Productive Cows as a Way to Improve Milk Production Technology
}

\author{
Dmitry Skachkov ${ }^{1}$, Vladimir Konvay ${ }^{1,2, *}$, Mikhail Zabolotnykh ${ }^{1}$ and Ekaterina Kornienko ${ }^{1}$ \\ ${ }^{1}$ Omsk State Agrarian University named after P.A. Stolypin, 1, Institutskaya Sq., Omsk, 644008, Russia \\ ${ }^{2}$ Omsk State Medical University (OSMU), 12, Lenin Str., Omsk, 644099, Russia
}

\begin{abstract}
The aim of this study was to study the molecular mechanisms of metabolic disorders that develop in highly productive cows. It was found that in the blood serum of animals with subclinical ketosis (SCK), which are in the eighth month of pregnancy, the concentration of $\beta$-hydroxybutyrate is increased, the content of iron, calcium and phosphorus is reduced, its iron-binding capacity against the background of the absence of phenomena of hepatocellular insufficiency, violation of the integrity of hepatocytes, hypoplastic anemia. The aggravation of ketoacidosis in fresh cows with rumen acidosis is facilitated by the development of carbohydrate deficiency in them, due to their increased oxidation in the reactions of anaerobic glycolysis, accompanied by excessive catabolism of purine mononucleotides to uric acid. Excessive lipid peroxidation of membrane structures under conditions of increased catabolism of purine nucleotides, along with the accumulation of acylglycerols in the liver caused by cobalamin deficiency, leads to the development of syndromes of impaired hepatocyte integrity and hepatocellular insufficiency. A decrease in the liver's ability to store iron ions and synthesize proteins that provide an increase in the iron-binding capacity of blood plasma in conditions of cobalamin deficiency contributes to the development of hypoplastic anemia not only in cows, but also in calves born from them.
\end{abstract}

\section{Introduction}

Modern dairy farming can be profitable only when using highly concentrated feeding, which allows getting high milk yields. At the same time, its use is often associated with a dysfunction of the digestive system organs, which have been adapted in ruminants for many millions of years to use cellulose as the main source of nutrition. It is important that the microflora inhabiting their organs of the digestive system not only hydrolyzes this polysaccharide to propionic acid, but also generates the derivatives of cobalalamin necessary for the assimilation of this acid by the body of ruminants. Excessive use of starch-rich feed leads to inhibition of the vital activity of these microorganisms [1]. The consequence of this may be not only a deep violation of the processes of energy supply, but also a decrease in the supply of the body with methyl groups participating in many vital processes $[2,3]$.

\section{Problem statement}

Metabolic disorders are a serious problem for dairy farming, manifested by decreased productivity and early culling of animals. Their consequence can be the development in highly productive cows of ketoacidosis, fatty liver infiltration, multiple organ failure, pathology of pregnancy and birth weakness. As a result, a significant part of animals do not live up to the age of 4-6th lactation, in which they could show maximum productivity, recoup the costs of growing and maintenance [4]. The pathogenesis of these conditions is not fully understood, which limits the development of new methods of diagnosing and treating them.

The purpose of this study was to study the mechanisms of metabolic disorders, in pregnant cows in which a form of subclinical ketosis (SCK) was diagnosed and in fresh animals, ketosis and rumen acidosis (K+RA) were revealed, as well as the development of methods for diagnosing pregnancy pathology to establish these conditions in cows.

\section{Materials and methods}

The research was carried out in different farms of the Omsk region, about 150 cows of black variegated and red steppe breed were examined. Of these, 37 conditionally healthy animals were isolated, 52 dry cows with subclinical ketosis and 61 new-calf animals with revealed ketosis and acidosis. Blood sampling from cows was carried out from the jugular vein. For biochemical studies, blood serum was used, which was obtained by centrifugation at $3000 \mathrm{rpm}$ for $10 \mathrm{~min}$. It was analyzed by unified methods of biochemical research using equipment from the Agrarian and Technological

\footnotetext{
* Corresponding author: vd.konvay@omgau.org
} 
Research Center for Collective Use and reagents from Hospitex (Switzerland, Italy). In the blood serum, the concentration of $\beta$-hydroxybutyrate, glucose, phosphatidylcholine, acylglycerols, total protein, albumin, globulins, cholesterol, iron, calcium, phosphorus, lactic acid, uric acid, total (TIBC) and latent iron binding capacity (LIBC), activity of lactate dehydrogenase, $\gamma$-glutamyl transferase and alkaline phosphatase by standardized biochemical methods [5]. The blood was studied for the number of erythrocytes, leukocytes in the Goryaev's chamber, the hematocrit and the hemoglobin content [6], and for the level of malondialdehyde in the erythrocytes [7]. The research results were processed statistically using the Student's test and nonparametric methods of mathematical analysis.

\section{Results and discussion}

From the data presented in the table, it can be seen that the concentration of $\beta$-hydroxybutyric acid in the blood of fresh cows with subclinical ketosis (SCK group) increases by $50.0 \%(\mathrm{P}=6.7)$, and in animals with ketosis and rumen acidosis (group $\mathrm{K}+\mathrm{RA}$ ), by $97.4 \%$ $(\mathrm{P}=5.9)$ compared to the control. This phenomenon can be associated with the progressive development of microflora, which inhibits the vital activity of microorganisms that break down cellulose to propionic acid and at the same time produce cobalamin [1].

Table 1. Biochemical parameters of blood plasma of conditionally healthy cows (control, C), with subclinical ketosis in the interlactation period (SCK) and fresh cows with ketosis and rumen acidosis $(\mathrm{K}+\mathrm{RA}), \mathrm{M} \pm \mathrm{m}, \mathrm{n}=15$

\begin{tabular}{|c|c|c|c|}
\hline Indicators & C & SCK & K+RA \\
\hline Glucose [mmol/l] & $4.3 \pm 0.3$ & $4.2 \pm 0.04$ & $3.9 \pm 0.5$ \\
\hline Lactic acid [mmol/1] & $0.56 \pm 0.04$ & $0.64 \pm 0.05$ & $0.82 \pm 0.07 *$ \\
\hline $\begin{array}{c}\beta \text {-hydroxybutyrate } \\
{[\mathrm{mmol} / \mathrm{l}]}\end{array}$ & $0.38 \pm 0.02$ & $0.57 \pm 0.02 *$ & $0.75 \pm 0.06^{*}$ \\
\hline Uric acid $[\mu \mathrm{mol} / \mathrm{l}]$ & $112 \pm 12$ & $114 \pm 15$ & $172 \pm 13^{*}$ \\
\hline $\begin{array}{l}\text { Malonic dialdehyde [u. } \\
\text { opt. dens./g erythr.] }\end{array}$ & $0.76 \pm 0.07$ & $0.72+0.06$ & $1.13 \pm 0.09 *$ \\
\hline Total protein [g/l] & $74.7 \pm 2.8$ & $79.1 \pm 1.4$ & $77.3 \pm 2.8$ \\
\hline Albumin $[\mathrm{g} / \mathrm{l}]$ & $37.2 \pm 0.31$ & $34.9 \pm 1.36$ & $33.2 \pm 0.49$ \\
\hline Globulins [g/l] & $37.5 \pm 2.6$ & $44.2 \pm 2.2$ & $44.1 \pm 3.1$ \\
\hline $\begin{array}{l}\text { Phosphatidylcholine } \\
{[\mathrm{mmol} / \mathrm{l}]}\end{array}$ & $10.3 \pm 1.2$ & $9.8 \pm 1.0$ & $6.9 \pm 0.6^{*}$ \\
\hline Acylglycerols [mmol/l] & $1.14 \pm 0.09$ & $1.12 \pm 0.12$ & $2.06 \pm 0.17 *$ \\
\hline Cholesterol [mmol/l] & $3.70 \pm 0.18$ & $4.90 \pm 0.83$ & $6.30 \pm 1.06^{*}$ \\
\hline TIBC $[\mu \mathrm{mol} / 1]$ & $65.2 \pm 3.7$ & $50.0 \pm 2.9^{*}$ & $48.7 \pm 2.8^{*}$ \\
\hline LIBC $[\mu \mathrm{mol} / \mathrm{l}]$ & $34.9 \pm 3.8$ & $29.0 \pm 3.9$ & $28.9 \pm 2.2 *$ \\
\hline Iron $[\mu \mathrm{mol} / \mathrm{l}]$ & $30.3 \pm 1.5$ & $21.0 \pm 2.4^{*}$ & $19.8 \pm 0.9 *$ \\
\hline Hemoglobin $[\mathrm{g} / \mathrm{l}]$ & $109 \pm 7$ & $103 \pm 9$ & $92 \pm 10^{*}$ \\
\hline Erythrocytes $\left[\mathrm{mln} / \mathrm{cm}^{3}\right]$ & $7.4 \pm 0.6$ & $6.8 \pm 0.5$ & $5.2 \pm 0.6^{*}$ \\
\hline Hematocrit [\%] & $38.3 \pm 2.7$ & $34.2 \pm 1.8$ & $31.3 \pm 2.2 *$ \\
\hline LDH [IU/l] & $2584 \pm 104$ & $2850 \pm 382$ & $2872 \pm 379$ \\
\hline GGT [IU/1] & $20.0 \pm 1.9$ & $25.8 \pm 1.3$ & $27.7 \pm 3.5^{*}$ \\
\hline Alkaline phosphatas [IU/L] & $122 \pm 17$ & $100 \pm 10$ & $97 \pm 12$ \\
\hline Calcium [mmol/l] & $2.76 \pm 0.10$ & $2.42 \pm 0.12$ & $2.22 \pm 0.05$ \\
\hline Phosphorus [mmol/1] & $1.80 \pm 0.09$ & $1.68 \pm 0.09$ & $1.54 \pm 0.08^{*}$ \\
\hline $\mathrm{Ca} / \mathrm{P}$ & $1.54 \pm 0.04$ & $1.45 \pm 0.08$ & $1.45 \pm 0.08^{*}$ \\
\hline
\end{tabular}

Note: $* \mathrm{P}<0.05$ (the difference is statistically significant

relative to the control group) reliability
Lack of vitamin B12 limits one of the reactions of further utilization of propionate, formed during the breakdown of cellulose by microorganisms of the rumen, - the conversion of methylmalonyl-CoA into succinyl-CoA. This reaction is catalyzed by methylmalonyl-CoA mutase, the coenzyme of which is adenosylcobalamin, a cobalamin derivative [8]. Succinyl-CoA, formed in this reaction, is necessary in ruminants for the constant "feeding" of the reactions of the Krebs cycle, especially in conditions of a deficiency of carbohydrates in the body [1].

As can be seen from the data presented in the table, such a deficit in the organism of cows of the K + RA group is pronounced, as evidenced by the tendency to a decrease in the concentration of glucose in the blood (by $9.3 \%$ compared with the control; $\mathrm{P}=0.7$ ). It can be associated with enhanced oxidation of this monosaccharide in the reactions of anaerobic glycolysis. The intensification of the latter in cows of the $\mathrm{K}+\mathrm{RA}$ group is evidenced by the increased content of lactic acid in the blood (by $46.4 \%$ in relation to the same indicator in the control; $\mathrm{P}=3.2$ ).

The reason for this increase is, apparently, the progression of the phenomena of hypoxia. It leads to inhibition of the generation of ATP in mitochondria, followed by an increase in the generation of this highenergy compound in the reactions of anaerobic glycolysis $[1,9]$. Since the latter are energetically insufficiently efficient (during anaerobic oxidation of one glucose molecule, only 2 ATP molecules are generated), their intensification leads to an increased consumption of carbohydrates with the subsequent development of their deficiency.

The lack of carbohydrates, in turn, leads to a compensatory increase in $\beta$-oxidation in the liver of free fatty acids to acetyl-CoA, followed by condensation of the latter to acetoacetic acid as a result of a reaction catalyzed by thiolase. Some of the acetoacetate molecules are reduced to $\beta$-hydroxybutyrate. Together, they are transferred by blood into the muscle and other tissues as part of ketone bodies, where they are decomposed back to acetyl-CoA, which is oxidized in the reactions of the Krebs cycle [1, 10].

Cobalamin deficiency in cows of the K + RA group leads not only to inhibition of the oxidation of ketone bodies in the Krebs cycle, but also limits the rate of transfer of the methyl group from methyltetrahydrofolic acid to homocysteine as a result of the reaction catalyzed by methyltetrahydrofolate: homocysteine methyltransferase. The coenzyme of the latter is methylcobalamin, also a cobalamin derivative. As a result, the production of methionine, which is a source of methyl groups in many biological processes, including in the reactions of choline and phosphatidylcholine biosynthesis, is disrupted $[8,11,12]$.

It proceeds in several stages: 1) replacement of a fatty acid located at the third carbon atom of acylglycerol with a phosphoric acid radical; 2) the addition of a choline residue to the latter and the formation of phosphatidylcholine (lecithin); 3) the entry of the latter from the liver into the blood, followed by the formation of transport lipoproteins. [2, 8, 12]. With a 
choline deficiency associated with a lack of cobalamin, the process of removing acylglycerols from the liver is limited and, as a result, fatty liver infiltration develops.
Replacement of the liver parenchyma with adipose tissue leads to disruption of various functions of this organ, including its ability to utilize lactate.

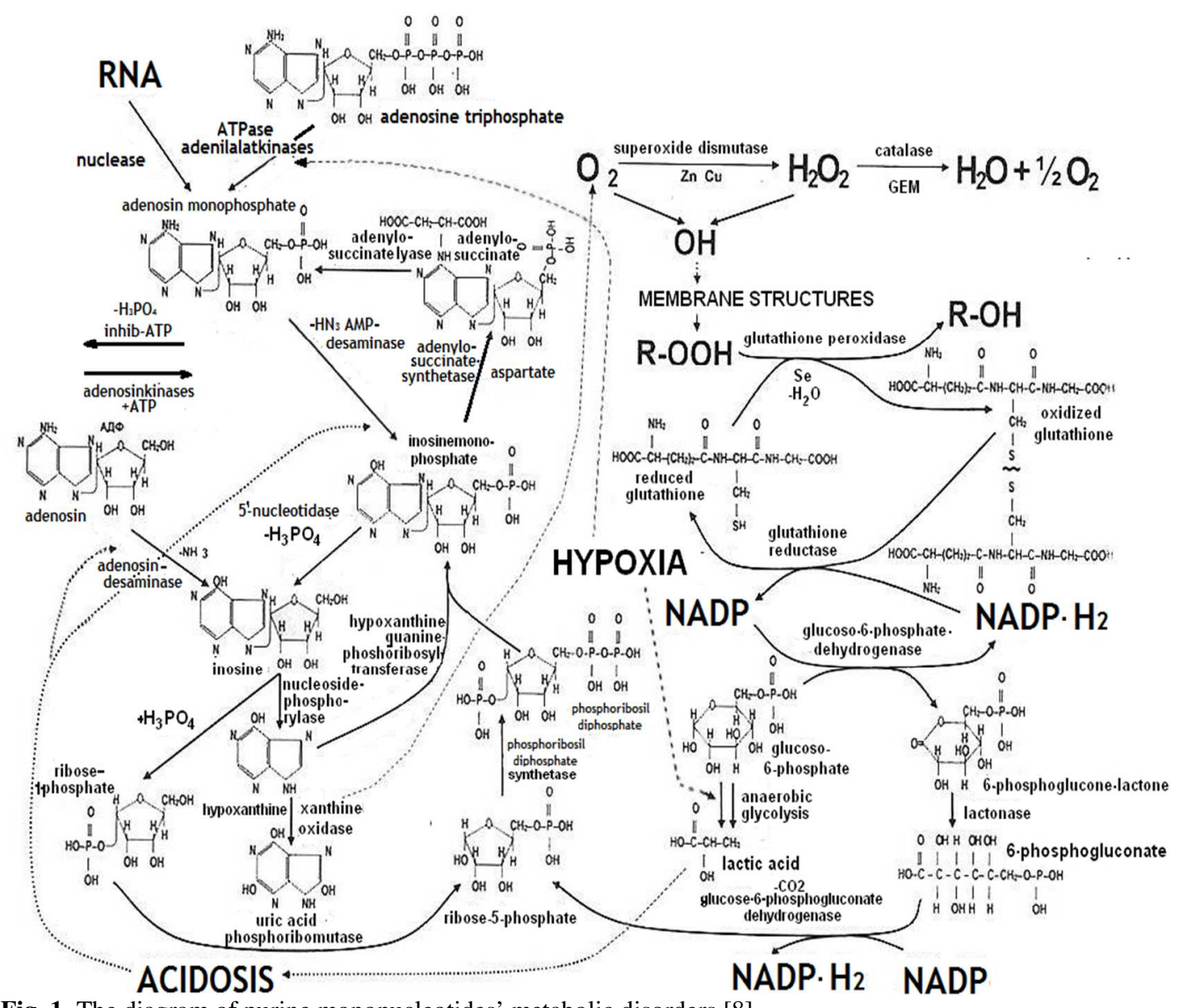

Fig. 1. The diagram of purine mononucleotides' metabolic disorders [8]

The intensification of this pathological process in the body of cows of the $\mathrm{K}+\mathrm{RA}$ group is evidenced by a decrease in blood plasma associated, evidently, with an increased concentration of phosphatidylcholine (by $33.0 \% ; \mathrm{P}=2.5$ ), involvement in the processes of excretion of acylglycerols from liver cells. An increase against the background of this level of acylglycerols in blood plasma (by $80.7 \%$; $\mathrm{P}=4.8$ ) indicates that the formation of transport lipoproteins in these animals is insufficiently efficient. Under these conditions, both in cows of the SCK group and in animals of the $\mathrm{K}+\mathrm{RA}$ group, signs of irritation syndrome of macrophage cells caused by exposure to lipids and other factors are expressed. This is expressed in a tendency to an increase in blood concentration of both total (by $3.5 \% ; \mathrm{P}=0.7$ ), globulins (by 17.6\%; $\mathrm{P}=1.6$ ), and cholesterol (by $70.3 \% ; \mathrm{P}=2.4)$. This syndrome in cows of the second of the named groups is combined, probably, with hepatocellular insufficiency.

It is expressed in the inhibition of the liver's ability to synthesize some specific proteins, in particular proteins that bind iron ions. The total and latent iron binding capacity of blood plasma proteins of animals of the $\mathrm{K}+\mathrm{RA}$ group is reduced, respectively, by $25.3 \%$ ( $\mathrm{P}=$ $3.6)$ and $17.2 \%(\mathrm{P}=1.4)$ in relation to the control. It can be assumed that due to the reduced content of these proteins, in the blood plasma, the iron ions received with the feed do not efficiently enter the liver cells and are deposited there. Their concentration in the blood rises above the permissible level and they are removed from the body by the kidneys.

This phenomenon, as well as the reduced ability of hepatocytes to deposit iron, leads to a decrease in the concentration of this substance in the blood serum (by $34.6 \%$ compared to the same indicator in the control; $\mathrm{P}=6.0$ ). This, along with a deficiency of cobalamin, which develops due to the effects on the body of the factors described above, leads to the development of anemia. Evidence of the presence of this disease in the blood of animals of the $\mathrm{K}+\mathrm{RA}$ group is a decrease in the concentration of hemoglobin, the number of erythrocytes and the hematocrit [respectively by $15.6 \%$ $(\mathrm{P}=1.4), 29.7 \%(\mathrm{P}=2.6)$ and $18,3 \%(\mathrm{P}=2.0)$ compared with similar indicators in the control].

Due to anemia, cows of the $\mathrm{K}+\mathrm{RA}$ group develop hemic hypoxia, followed by impaired oxygen delivery to the tissues, leading to inhibition of ATP generation by enzymes of the mitochondrial respiratory chain. As a result, there is a compensatory increase in the production of this high-energy compound in the reactions of anaerobic glycolysis, followed by an increase in the blood plasma concentration of the final product of this 
metabolic pathway-lactic acid. Its concentration in the blood of cows of the $\mathrm{K}+\mathrm{RA}$ group exceeds the same indicator in control animals by $46.4 \%(\mathrm{P}=3.2)$. This, along with the acidification of tissues by ketone bodies, contributes to the development of acidosis.

Under conditions of acidification of the contents of cells with lactate and ketone bodies, the catabolism of purine mononucleotides intensifies. This process begins with an increase in the activity of adenylate deaminase and adenosine deaminase, which catalyze the key reactions of purine catabolism. It is known that these enzymes are activated under conditions of acidification of tissue contents with acidic equivalents [13, 14]. This promotes the breakdown of AMP to hypoxanthine. The latter, under conditions of insufficiently effective reutilization as a result of a reaction catalyzed by hypoxanthine-guanine phosphoribosyl transferase, is oxidized to uric acid as a result of a reaction involving xanthine oxidase [15].

The level of this metabolite in the blood plasma of cows of the $\mathrm{K}+\mathrm{RA}$ group exceeds that in control animals by $53.6 \%(\mathrm{P}=3.3)$. At the same time, xanthine oxidase, which has high activity in the endothelium of blood vessels, especially in the liver, kidneys and mucous membrane of the small intestine [15], produces active oxygen metabolites that, under conditions of inhibition of the function of the antioxidant system, in particular, a decrease in the activity of antiradical defense enzymes, enhance lipoperoxidation of membrane cell structures. An increase in the intermediate product of its malondialdehyde in erythrocytes is evidence of the intensification of this process in the organism of cows of the $K+$ RA group [16]. The content of this substance in the erythrocytes of animals of the $\mathrm{K}+\mathrm{AR}$ group exceeds the level of malondialdehyde in these cells in control animals by $48.7 \%(\mathrm{P}=3.2)$.

Excessive lipid peroxidation of membrane structures is facilitated by the intensification of purine metabolism, coupled with an increase in lipid peroxidation of membrane structures (Fig. 1). Along with the accumulation of acylglycerols in the liver, caused by cobalamin deficiency, it leads to the development of syndromes of violation of the integrity of hepatocytes and hepatocellular insufficiency. This is reflected in an increase in the activity of enzymes in the blood contained in cells. In the blood plasma of these animals, the activity of $\gamma$-glutamyltransferase is increased, which exceeds that in control animals by $38.5 \%(\mathrm{P}=2.0)$. At the same time, there is a tendency towards an increase in the activity of lactate dehydrogenase in this biological fluid (by $11.1 \% ; \mathrm{P}=0.73$ ).

It can be assumed that under conditions of intensification of the production of active oxygen metabolites by xanthine oxidase, lipoperoxidation of arachidonic acid residues in phospholipid molecules of membrane structures is enhanced. This leads to a violation of the permeability of the latter with a subsequent increase in the flow of enzymes into the blood. These enzymes are found in various organs. Nevertheless, it can be assumed that the increase in their activity is mainly associated with damage to the liver, in which the highest activity of xanthine oxidase $[15\}$.

Another organ highly susceptible to damage by active oxygen metabolites generated by xanthine oxidase and other sources is, obviously, the parathyroid glands. They provide incretion of parathormone and thyrocalcitonin, which regulate the level of calcium in the blood. The content of the latter, as well as the inorganic phosphate functionally related to it, in the blood plasma of cows of the $\mathrm{K}+\mathrm{RA}$ group is reduced by $19.6 \%(\mathrm{P}=4.8)$ and $14.4 \%(\mathrm{P}=2.2)$, respectively, compared with the control.

A decrease in the intake of calcium ions into the blood associated with damage by active oxygen metabolites of the cells of the small intestine and kidneys can play a certain role in reducing the level of these indicators. Due to a decrease in the level of these substances in the blood, calcium and phosphorus are washed out of the bone tissue, followed by a violation of its structure. This is reflected in the fact that cows of the $\mathrm{K}+\mathrm{RA}$ group often develop osteodystrophy, more often in the form of osteomalacia.

\section{Conclusion}

Thus, it follows from the data obtained that in the blood plasma of cows with subclinical ketosis (SCK), which are in the eighth month of pregnancy, the concentration of $\beta$-hydroxybutyrate is increased, the content of iron, calcium and phosphorus is reduced, its iron binding capacity is reduced against the background of the absence of phenomena of hepatocellular insufficiency and violation of the integrity of hepatocytes and hypoplastic anemia.

The ketoacidosis in fresh cows with rumen acidosis is aggravated by the developed deficiency of carbohydrates caused by their increased oxidation in the reactions of anaerobic glycolysis accompanied by excessive catabolism of purine mononucleotides to uric acid and coupled with increased lipid peroxidation.

Excessive lipid peroxidation of membrane structures under conditions of increased catabolism of purine nucleotides, along with the accumulation of acylglycerols in the liver caused by cobalamin deficiency, leads to the development of syndromes of impaired hepatocyte integrity and hepatocellular insufficiency.

This is reflected in a decrease in the liver's ability to store iron ions and synthesize proteins that increase the iron-binding capacity of blood plasma. In conditions of concomitant deficiency of cobalamin, this leads to the development of hypoplastic anemia not only in mothers, but also in calves born from them.

\section{References}

1. V. Konvay, M. Zabolotnykh, Metabolic disorders in highly productive cows, Bull. of Omsk State Agrar. Univ., 3(27), 130-136 (2017)

2. D. Skachkov, M. Zabolotnykh, V. Conway, Oxidation processes in the organisms of calves born 
by high-yelding cows, Int. J. Pharm. Res., 10(4), 755 (2018)

3. W. Marshall, S. Bangert, Clinical biochemistry (Binom, Moscow, 2015), 408 p.

4. I. Kalyzhniy, I. Stepanov, A. Shimanova, D. Markova, M. Kenzhegaliyeva, Hepatosisin HighYelding Cowsof Holstein Breed, Adv. in animal and veter. Sci., 7, 1-7 (2019)

5. I.P. Kondrakhin, Methods of veterinary clinical laboratory diagnostics (Kolos, Moscow, 2004), $230 \mathrm{p}$.

6. E. Van Kampen, W. Zijstra, The Haemoglobin Clin, Chim., 6(4), 538-639 (1961)

7. N. Stalnaya, T. Garishvili, Method for determination of malondialdehyde using thiobarbituric test (Modern methods of biochem., Moscow, 1997), pp. 66-68.

8. G. Stangl, Evaluation of the cobalt requirement of beef cattle based on vitamin B12, folate, homocysteine, and methylmalonic acid, Br. J. Nutr., 84, 645-653 (2000)

9. D. Markova, I. Kalyuzhny, S. Baizuldinov, Hematological parameters in cows with metabolic disorders during the adaptation period, Bull. of the Kursk State Agricult. Acad., 4, 106-111 (2018)
10. R. Murray, D. Grenner, P. Meyes, W. Rodwell, Human Biochemistry, In 2 volumes (World, Moscow, 1993), vol. 1, 382 p.; vol. 2, 414 p.

11. S. Paudyal, F. Maunsell, J. Richeson, C. Risco, P. Donovan, Peripartal rumination dynamics and health status in cows calving in hot and cool seasons, J. Dairy Sci., 99(11), 9057-9068 (2016)

12. M. Sheehy, A. Fahey, S. Aungier, F. Carter, M. Crowe, F. Mulligan, A comparison of serum metabolic and production profiles of dairy cows that maintained or lost body condition 15 days before calving, J. Dairy Sci., 100(1), 536-547 (2017)

13. J. Cory, Adenosine deaminase, Biochem., 4, 17291731 (1965)

14. Y. Lee, Adenylic acid deaminase, J. Biol. Chem., 227(5), 987-993 (1957)

15. D. Parks, D. Granger, Xanthine oxidase: biochemistry, distribution and physiology, Acta physiol. scand., 126(548), 87-89 (1986)

16. S. Babukhin, V. Avdeenko, I. Kalyuzhny, A. Molchanov, S. Tresnitsky, Disruption of metabolic processes in the body of pregnant cows during the development of subclinical ketosis, Agrar. Sci. J., 11, 6-11 (2016) 